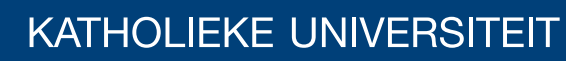 \\ LEUVEN
}

\section{Faculty of Business and Economics}

6 HOIP SRMHGMP HZZ LQRZV QQYHKIFOILRXUQJISLREOP V

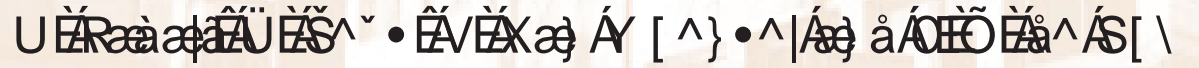

DEPARTMENT OF DECISION SCIENCES AND INFORMATION MANAGEMENT (KBI) 


\title{
Self-Imposed Time Windows in Vehicle Routing Problems
}

\author{
October 21, 2010
}

\begin{abstract}
We speak of Self-Imposed Time Windows (SITW) when a logistics service provider quotes a delivery time window to his customer. Once this time window is communicated, the company strives to respect it as well as possible. We incorporate these SITW within the framework of the Vehicle Routing Problem (VRP). Essential to SITW is the fact that the time window is determined by the carrier company and not by the customer. The resulting VRP-SITW is inherently different from the well-studied VRP with Time Windows (VRPTW) in that in the latter problem the time windows are exogenous constraints imposed by the customers. The second important element of the problem studied in this paper is the uncertainty in the travel times. The basic mechanism of dealing with this uncertainty is the allocation of time buffers throughout the routes, which absorb disruptions. We propose a heuristic solution approach combining an LP model and a local search heuristic. A tabu search heuristic assigns customers to vehicles and establishes the order of visit of the customers per vehicle. Detailed timing decisions are subsequently generated by the LP model, whose output also guides the local search in a feedback loop. We test our algorithm on a number of benchmark instances for the VRP and VRPTW. We highlight the costs involved in integrating SITW with the VRP and we underline the advantages to the carrier company of SITW when compared to VRPTW.
\end{abstract}

Keywords: routing; vehicle routing problem; vehicle scheduling; disruptions in travel times; tabu search; linear programming. 


\section{Introduction}

Many small-package shipping companies provide their customers with a time window for delivery and display this in their online tracking system. UPS, for instance, shows information on the delivery time window of orders for DELL computers. Obviously, once a time window is quoted to the customer, the carrier company wants to service the client within this window and so this should be reflected in the carrier's routing decisions. The described environment is clearly distinct from both the classic Vehicle Routing Problem (VRP) as well as from the VRP with Time Windows (VRPTW). It is different from the VRP since the VRP objective is to minimize the operational costs (e.g., distances or travel times (Laporte, 1992)). The VRPTW, on the other hand, does consider time windows but assumes they are exogenous, i. e., imposed by the customer (Bräysy and Gendreau, 2005a,b). As a consequence, the VRPTW imposes restrictions on the specific arrival times at each customer, while maintaining the objective of minimizing operational costs.

Our problem at hand considers time windows but treats them as endogenous to the VRP model. Specifically, the carrier company assigns customers to vehicles, sequences the customers allocated to each vehicle, and sets the time windows in which it plans to serve the customers. In the remainder of this paper, we will refer to the described problem as the Vehicle Routing Problem with Self-Imposed Time Windows (VRP-SITW). The term 'self-imposed' refers to the fact that the carrier company selects the time windows by itself, independently of the customer. Once the time windows are quoted to the customer, however, the customer should be serviced within the window. As such, the VRP-SITW conceptually lies in between the VRP and the VRPTW. We assume that service cannot start before the time window, leading to waiting in case of early arrivals. Furthermore, late arrivals are permitted but penalized proportionally to their tardiness. Drivers have a fixed shift length and are paid a fixed amount per day. Finally, disruptions in traveling time may occur between each two customers. This mainly reflects accidents, weather condition, vehicle breakdown or road works. One natural way to protect schedules against this uncertainty 
is to include time buffers (see, for instance, Hopp and Spearman (1996) for a similar logic in a production environment). Inspired by the scheduling literature, we propose a buffer allocation model that inserts slack time into the schedule to cope with possible delays. Our solution framework relies on the tabu search heuristic for assigning customers to routes and for the sequencing of each route. The actual evaluation of the target function is achieved by solving the resulting buffer allocation model to optimality for each route separately; this sub-problem is a linear programming problem. In the terminology of Puchinger and Raidl (2005), our hybrid algorithm is collaborative, since there is a clear hierarchy between the two phases. Examples of earlier works that combine local search with LP are Finke et al. (2007), where job-machine allocation is performed via tabu search while an LP model is used for inserting buffers in between jobs. Flisberg et al. (2009) solve a VRPTW via tabu search based on the input of an LP that defines origins and destinations for full truckloads.

The parallelism between vehicle routing and production scheduling is highlighted by Gendreau et al. (1995), who study single-vehicle routing and scheduling to minimize the number of delays. Given a deadline for servicing each customer, the objective is to minimize the number of late deliveries. The problem is equivalent to single-machine scheduling with sequence-dependent setup times to minimize the number of tardy jobs. The scheduling aspect is fundamental in Mitrović-Minć and Laporte (2004), in the context of dynamic pickup and delivery with time windows. The authors first solve the routing component and then look into the scheduling component. Four waiting strategies are presented and assessed based on the distance along with the number of vehicles required. Xiang et al. (2008) study the dynamic dial-a-ride under various types of uncertainty. They propose several scheduling strategies for handling dynamic events, accounting for a fixed duration and overtime costs in the case of exceeding the shift length. Our problem VRP-SITW differs from the above literature in that customer demand is known in advance. Stochastic travel times in VRP are investigated in Laporte et al. (1992), where vehicles incur penalties for exceeding a limit on the route duration. Li et al. (2010) examine VRPTW with stochastic travel and service 
times. Their model also includes overtime costs for exceeding route duration and soft time windows; the actual penalties are computed by means of simulation.

The main contributions of this paper are threefold: (1) we describe the new yet practical setting of SITW in vehicle routing; (2) we describe how a VRP with SITW and stochastic travel times can benefit from time buffers; and (3) we develop a hybrid LP / tabu search algorithm for producing high-quality solutions. Our aim is to construct a stable a priori plan that best copes with disruptions; in other words, a solution is generated at the start of the planning horizon and does not require further optimization during its implementation.

The remainder of the paper is organized as follows. We provide a number of definitions and a detailed problem statement in Section 2. Our solution procedure is described in Section 3. The computational experiments are presented and discussed in Section 4. Finally, in Section 5, we highlight the main results and indicate directions for future research.

\section{Description of VRP-SITW}

Consider a set of $N$ customers with a fleet of $K$ identical vehicles. Each customer $i$ has a demand $q_{i}$ and is to be serviced by a single vehicle. The logistics network is represented by a complete directed graph $G=(V, A)$, with $V=\{0,1, \ldots, N\}$ the set of vertices and $A$ the set of directed links. The vertex 0 denotes the depot; the other vertices of $V$ represent the customers. The non-negative weight $d_{i j}$ associated with each arc $(i, j)$ represents the distance from $i$ to $j$. Each vehicle must start and end its route at the depot, the total demand on each route cannot exceed the vehicle capacity $Q$ and each customer should be visited exactly once. The objective of the VRP is to construct routes that bring the total travel time of the vehicles to a minimum. The VRP-SITW entails the same elements as the VRP but with a number of additional parameters. Below, we first give a general description of the objective function (Section 2.1). Subsequently, we provide we elaborate on the SITW model and on the way in which stochasticity is captured (in Sections 2.2 and 2.3, respectively). 


\subsection{Objective function}

The objective function of the VRP-SITW consists of three parts. The first part is the travel cost, which captures the vehicle operating costs such as fuel costs. The second part of the objective function is a tardiness penalty, which represents the desire to respect the quoted time windows as well as possible. A 'railroad-scheduling approach' is adopted: the lower bound of the time window is the earliest starting time of the service. Arrival before the scheduled window is not penalized, since the driver cost is presumed to be fixed. Arrival after the time window, however, leads to a penalty proportional to the tardiness. The third component of the objective function is an overtime penalty. We suppose that the drivers are paid a fixed amount for a shift with fixed duration; if this duration is exceeded then overtime penalties are due.

In the optimal solution to the VRP-SITW, the travel time will never be less than for the associated VRP instance since the latter has neither tardiness nor overtime penalties. The travel time in optimal solutions to VRP-SITW and VRPTW is in principle incomparable, since the fixed time windows are relaxed in the former but there are extra penalties in the objective. The computational experiments described in Section 4 indicate that the VRPSITW leads to less travel time in most of the instances studied, presumably because the time windows are decision variables rather than constraints. With travel costs only, the VRP-SITW is equivalent to the VRP and is thus NP-hard.

A solution to the VRP-SITW is a set of routes $Z=\left\{R_{1}, R_{2}, \ldots, R_{|Z|}\right\}$ with $|Z| \leq K$.

Each route $R_{r}(r=1, \ldots,|Z|)$ is a vector $(0, i, j, \ldots, 0)$ whose components are elements of $V$, specifying which clients (vertices) will be visited by the vehicle following the route, and in which order. Each route begins and ends at the depot (vertex 0) and each vertex different from 0 belongs to exactly one route. We say that $i \in R_{r}$ if the vertex $i \in V$ is part of route $R_{r} \in Z$ and $(i, j) \in R_{r}$ if $i$ and $j$ are two consecutive vertices in $R_{r}$. The objective function 
for the VRP-SITW is then

$$
F(Z)=\Omega(Z)+\sum_{R_{r} \in Z} \Theta\left(R_{r}\right)
$$

with $\Omega(Z)$ the total travel cost associated with solution $Z$ and $\Theta\left(R_{r}\right)$ representing the overtime and tardiness penalties of route $R_{r}$. The travel cost is defined as follows:

$$
\Omega(Z)=c \sum_{R_{r} \in Z} \sum_{(i, j) \in R_{r}} d_{i j}
$$

with $c$ the cost of traveling one unit of distance. The penalties of each route are evaluated by solving a buffer allocation problem, which is described in Section 2.2.

\subsection{Self-imposed time windows}

Each route $R_{r}$ consists of a set of $n_{r}$ customers. For convenience, when referring to one specific route, we relabel the customers in ascending order: $R_{r}=\left(0,1,2, \ldots, n_{r}, n_{r}+1\right)$, where the depot corresponds with $0 \equiv n_{r}+1$. The distance $d_{i, i+1}$ between consecutive nodes $i$ and $i+1$ in the route is written as $d_{i}$. A schedule for route $R_{r}$ is an $\left(n_{r}+2\right)$-vector $\mathbf{s}=\left(s_{0}, s_{1}, \ldots, s_{n_{r}+1}\right)$, specifying a departure time $s_{i}$ from each node $i \in R_{r}$. The shift length is the time interval $\left[s_{s}, s_{e}\right]$, implying that $s_{s} \leq s_{0}$. Each customer $i \in R_{r} \backslash\left\{0, n_{r}+1\right\}$ has a time-window length $W_{i}$ within which the arrival of the vehicle is desired. The carrier company communicates time windows to its customers based on the schedule s. Each node $i \in R_{r}$ also has a standard service time $u_{i}$, e. g., for load/unload activities. We assume that a vehicle will never leave a customer earlier than scheduled. The left bound of the time window is then $s_{i}-u_{i}$, as this constitutes an earliest starting time for the servicing operations. An illustration is provided in Figure 1. The service times $u_{0}$ and $u_{n_{r}+1}$ at the depot are set to zero.

During the realization of this baseline schedule, disruptions might occur. We examine disruptions corresponding with an increase in the travel time $d_{i}$ between customers $i$ and $i+1$. The length $L_{i}$ of this delay is a random variable, which is modeled by means of 
discrete scenarios; a similar choice in a machine-scheduling context is made by, e.g., Daniels and Carrillo (1997), Daniels and Kouvelis (1995), Kouvelis et al. (2000), and Kouvelis and $\mathrm{Yu}$ (1997). Specifically, we let $L_{i}$ denote the increase in $d_{i}$ if $i$ is 'disrupted', which takes place with probability $p_{i}$. The variable $L_{i}$ is discrete with probability-mass function $g_{i}(\cdot)$, which associates non-zero probability with positive values $l_{i k} \in \Psi_{i}$, where $\Psi_{i}$ denotes the set of disruption scenarios for $d_{i}$, so $\sum_{k \in \Psi_{i}} g_{i}\left(l_{i k}\right)=1$. We use $g_{i k}$ as shorthand for $g_{i}\left(l_{i k}\right)$; the disruption lengths $l_{i k}$ are indexed from small to large for a given $i$. The realization of $L_{i}$ becomes known only when arc $(i, i+1)$ is traversed. The actual departure time at customer $i$ is denoted by $s_{i}^{a}(\mathbf{s})$; this is a random variable that is dependent on the schedule $\mathbf{s}$ (in the remainder of the article, we omit the argument $\mathbf{s}$ when there is no danger of confusion). The value $s_{i}-u_{i}$ is a lower bound on the starting time of the client's service. This socalled railroad-scheduling approach implies that $s_{i} \leq s_{i}^{a}, \forall i \in R_{r}$, and guarantees that the actual schedule will strictly copy the baseline schedule if no disruptions occur. In effect, the scheduled times become 'release dates' for departure times $s_{i}^{a}$ from each customer $i \in R_{r}$ :

$$
\begin{aligned}
& s_{0}^{a}=s_{0} \\
& s_{i}^{a}=\max \left\{s_{i} ; s_{i-1}^{a}+d_{i-1}+L_{i-1}+u_{i}\right\}, \quad i=1, \ldots, n_{r}+1 .
\end{aligned}
$$

Arrival prior to $s_{i}-u_{i}$ is not penalized. With arrival later than $s_{i}-u_{i}+W_{i}$, however, we associate a cost proportional to the tardiness: a non-negative integer penalty $t_{i}$ is incurred

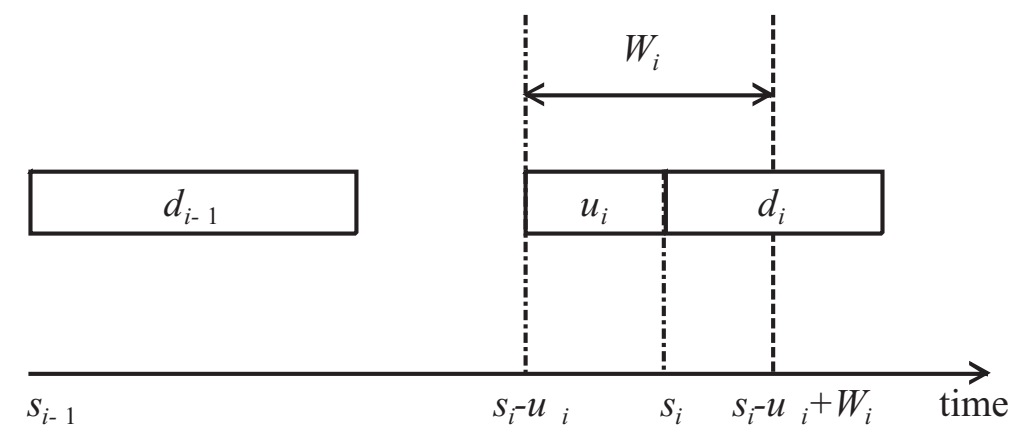

Figure 1: Illustration of a time window at customer $i$ 
per unit-time delay. The value $t_{n_{r}+1}$ is the cost for arriving late at the depot at the end of the tour.

We assume that the driver receives a fixed payment for the shift, which ends at $s_{e}$. Arrival after the end of the shift incurs an overtime penalty $b$ per time unit. We can now elaborate the penalty term $\Theta(\cdot)$ in Equation (1). For a given route $R_{r}, \Theta\left(R_{r}\right)$ consists of two components, namely the expected delay costs at customers and at the depot on the one hand, and the expected overtime penalty on the other hand. Specifically,

$$
\Theta\left(R_{r}\right)=\sum_{i \in R_{r} \backslash\{0\}} t_{i} \mathbb{E}\left[\max \left\{0 ; s_{i}^{a}(\mathbf{s})-\left(s_{i}-u_{i}+W_{i}\right)\right\}\right]+b \mathbb{E}\left[\max \left\{0 ; s_{n_{r}+1}^{a}-s_{e}\right\}\right]
$$

with $\mathbb{E}[\cdot]$ the expectation operator (note that $\mathbf{s}$ is actually also a parameter to $\Theta(\cdot)$ ). In the following subsection, we outline the disruption model in detail.

\subsection{Modeling disruptions}

When the durations are independent, little less is possible for objective-function evaluation than to consider all $\prod_{i \in R_{r} \backslash\left\{n_{r}+1\right\}}\left(\left|\Psi_{i}\right|+1\right)$ possible combinations of duration disruptions. This was the motivation in a scheduling context in Herroelen and Leus (2004); Leus and Herroelen (2005, 2007); Ballestín and Leus (2008) to develop a model that considers only the main effects of the separate disruption of each of the individual jobs rather than all possible disruption interactions. Computational results in the aforementioned scheduling applications show that the resulting model is quite robust to variations in the actual number of disrupted jobs. In the context of time-dependent VRP with service disruptions, Jabali et al. (2009) also focus on the effects of single disruptions. We make a similar assumption in this paper: our model assumes that exactly one leg will suffer a disruption from its baseline duration. The underlying practical motivation is that we should only optimize for one 'inconvenience' per day, as it would be very difficult to protect from multiple disruptions at multiple places at multiple times. The resulting restricted model is useful when disruptions 
are sparse and spread over time so that the number of interactions is limited.

For a given route $R_{r}$ we distinguish between two situations: either no leg in $R_{r}$ is disturbed, or a single leg is disturbed in $R_{r}$. Let $\zeta$ denote the overtime for $R_{r}$ when no leg is disturbed (tardiness penalties are irrelevant if no leg is disturbed). The total penalty $\Theta\left(R_{r}\right)$ consists of two components, namely the expected delay costs at customers and at the depot on the one hand, and the expected overtime penalty on the other hand. Specifically, for a given route $R_{r}$, under the one-disruption assumption and with $s_{i-1}+d_{i-1}+u_{i} \leq s_{i}$ for all $i>0$, the relevant penalty term in (2) can be written as

$$
\Theta\left(R_{r}\right)=\sum_{i=0}^{n_{r}} \sum_{j=i+1}^{n_{r}+1} \sum_{k=1}^{\left|\Psi_{i}\right|} p_{i} g_{i k} t_{j} \Delta_{i j k}+b \sum_{i=0}^{n_{r}} \sum_{k=1}^{\left|\Psi_{i}\right|} p_{i} g_{i k} \Lambda_{i k}+b\left(1-\sum_{i=0}^{n_{r}} p_{i}\right) \zeta
$$

In this expression,

$$
\begin{gathered}
\Delta_{i j k}=\max \left\{0 ; s_{i}+d_{i}+l_{i k}+\sum_{m=i+1}^{j-1}\left(u_{m}+d_{m}\right)-s_{j}+u_{j}-W_{j}\right\}, \\
i \in R_{r} \backslash\left\{n_{r}+1\right\} ; j \in R_{r} \backslash\{0\} ; i<j ; k \in \Psi_{i}, \\
\Lambda_{i k}=\max \begin{cases}0 & \left.; \quad s_{n_{r}+1}+\Delta_{i, n_{r}+1, k}-s_{e}\right\}, \quad i \in R_{r} \backslash\left\{n_{r}+1\right\} ; k \in \Psi_{i}\end{cases}
\end{gathered}
$$

and

$$
\zeta=\max \left\{0 ; \quad s_{n_{r}+1}-s_{e}\right\}
$$

Remember that $p_{i}$ represents the probability that $d_{i}$ is the unique disrupted value. The variable $\Delta_{i j k}$ represents the tardiness at client $j$ due to a disruption according to scenario $k$ of $d_{i}$, which is equal to zero or to the disruption length of $i$ minus the buffer size in place between the customers $i$ and $j$, whichever is larger. The term $\sum_{m=i+1}^{j-1}\left(u_{m}+d_{m}\right)$ is the service and travel time for the customers between $i$ and $j$. Similarly, $\Lambda_{i k}$ is the overtime resulting from a disruption at customer $i$ by scenario $k$. The overtime is zero in case of arrival at the depot before the shift end $s_{e}$, and equal to the realized arrival time minus $s_{e}$ 
otherwise. Thus, $\zeta$ is zero in case of arrival at the depot before the shift end. The probability that a route is not disturbed is $\left(1-\sum_{i=0}^{n_{r}} p_{i}\right)$.

\section{A hybrid solution procedure}

Our solution method for the VRP-SITW proceeds in two stages: first routing and then scheduling. The assignment of customers to vehicles and the sequencing of customers are

done in stage 1; this stage uses tabu search. Iteratively, the routes generated by the tabu search are then scheduled in the second stage, where we use linear programming to solve the sub-problem to optimality under the one-disruption assumption. We say that our solution procedure is 'hybrid' due to the combined use of a meta-heuristic and an exact optimization routine. Below, we first describe the lower-level scheduling problem in Section 3.1, followed by the tabu search procedure (Section 3.2).

\subsection{Scheduling and buffer insertion}

For a given route $R_{r}$, the linear program below produces an optimal schedule, conditional on exactly one leg being disrupted. Buffer sizes are implicit from the resulting schedule.

$$
\Theta\left(R_{r}\right)=\min \quad \sum_{i=0}^{n_{r}} \sum_{j=i+1}^{n_{r}+1} \sum_{k=1}^{\left|\Psi_{i}\right|} p_{i} g_{i k} t_{j} \Delta_{i j k}+b \sum_{i=0}^{n_{r}} \sum_{k=1}^{\left|\Psi_{i}\right|} p_{i} g_{i k} \Lambda_{i k}+b\left(1-\sum_{i=0}^{n_{r}} p_{i}\right) \zeta
$$


subject to

$$
\begin{aligned}
s_{i-1}+d_{i-1}+u_{i} & \leq s_{i} \quad i \in R_{r} \backslash\{0\} \\
s_{0} & \geq s_{s} \\
s_{i}+d_{i}+l_{i k}+\sum_{m=i+1}^{j-1}\left(u_{m}+d_{m}\right) & \leq s_{j}-u_{j}+W_{j}+\Delta_{i j k} \\
i & \in R_{r} \backslash\left\{n_{r}+1\right\} ; j \in R_{r} \backslash\{0\} ; i<j ; k \in \Psi_{i} \\
s_{n_{r}+1}+\Delta_{i, n_{r}+1, k}-s_{e} & \leq \Lambda_{i k} \quad i \in R_{r} \backslash\left\{n_{r}+1\right\} ; k \in \Psi_{i} \\
\zeta & \geq s_{n_{r}+1}-s_{e} \\
\text { all } \Delta_{i j k} & \geq 0 ; \text { all } s_{i} \geq 0 ; \text { all } \Lambda_{i k} \geq 0 ; \zeta \geq 0
\end{aligned}
$$

Constraints (3) can be viewed as precedence constraints: the scheduled departure time $s_{i}$ from customer $i$ is at least equal to the departure time of its predecessor $s_{i-1}$ augmented with the distance $d_{i-1}$ and the service time $u_{i}$. This implies that the buffer between customers $i-1$ and $i$ is $s_{i}-\left(s_{i-1}+d_{i-1}+u_{i}\right)$. Constraint (4) ensures that the scheduled departure time from the depot does not precede the shift's start time $s_{s}$. Constraints (5), (6) and (7) determine the delay terms $\Delta_{i j k}, \Lambda_{i k}$ and $\zeta$, respectively, as described in Section 2.3.

\subsection{Tabu search for the VRP-SITW}

Tabu search has been widely used for solving the VRP, see for example Gendreau et al. (1994, 1996); Hertz et al. (2000). Furthermore, it has been extensively used to solve VRPTW as well, examples can be found in Garcia et al. (1994); Taillard et al. (1997). Thus, adopting the tabu search heuristic comes as a natural choice also for the VRP-SITW. Our tabu search procedure generates a set of routes that still need to be scheduled using the lower-level LP

described in Section 3.1. The procedure iteratively scans the members of a neighborhood of the current solution to evaluate possible improvements in the objective function. Due to our bi-level approach, the evaluation of each neighborhood solution requires a separate 
LP run, which, if performed to optimality, would require enormous computation times. We have therefore opted for approximations of these optimal overtime and tardiness penalties to guide the tabu search in selecting the best move in its current neighborhood. Once a move is selected, its exact target function is computed by invoking the LP model for the changed route or routes, leading to a new optimal schedule.

The overall procedure is described in pseudo-code as Algorithm 1. We adopt three different criteria $C_{1}, C_{2}$ and $C_{3}$ for choosing a move; these will be described in detail below. The tabu search procedure is run consecutively with each of the three criteria. The initial solution $Z_{0}$ is the output of the nearest neighbor heuristic for each of the three criteria. Feeding the best-found solution of $C_{1}$ into the run for $C_{2}$ and for $C_{2}$ into $C_{3}$ has been tested, together with many variations of the order of the three criteria, but this did not lead to better results. For each customer $i \in V$, we construct 2-opt* (Potvin and Rousseau, 1995) and Oropt (Or, 1976) neighborhoods for the $\eta$ nodes closest to $i$. A chosen move is declared tabu for the next $\kappa$ iterations. The process iterates until a maximum number of non-improving moves is reached.

In line with Gendreau et al. (1994), diversification of the search is achieved by allowing demand-infeasible solutions (i.e., routes with total demand exceeding the vehicle capacity). Such infeasible solutions are penalized in proportion to their capacity violation by means of the following composite objective function, which replaces $\Omega(Z)$ :

$$
\Omega_{2}(Z)=\Omega(Z)+w \sum_{R_{r} \in Z}\left[\left(\sum_{i \in R_{r}} q_{i}\right)-Q\right]^{+}
$$

In Equation (9) each unit of excess demand is penalized by a factor $w$. This excess penalty $w$ is decreased by multiplication with a factor $\nu$ after $\phi$ consecutive feasible moves. Similarly, $w$ is increased (multiplied by factor $\nu^{-1}$ ) after $\phi$ infeasible iterations.

Below, we describe the three criteria that allow avoiding the use of the LP model for each candidate solution and lead to computationally efficient move selection procedures. 


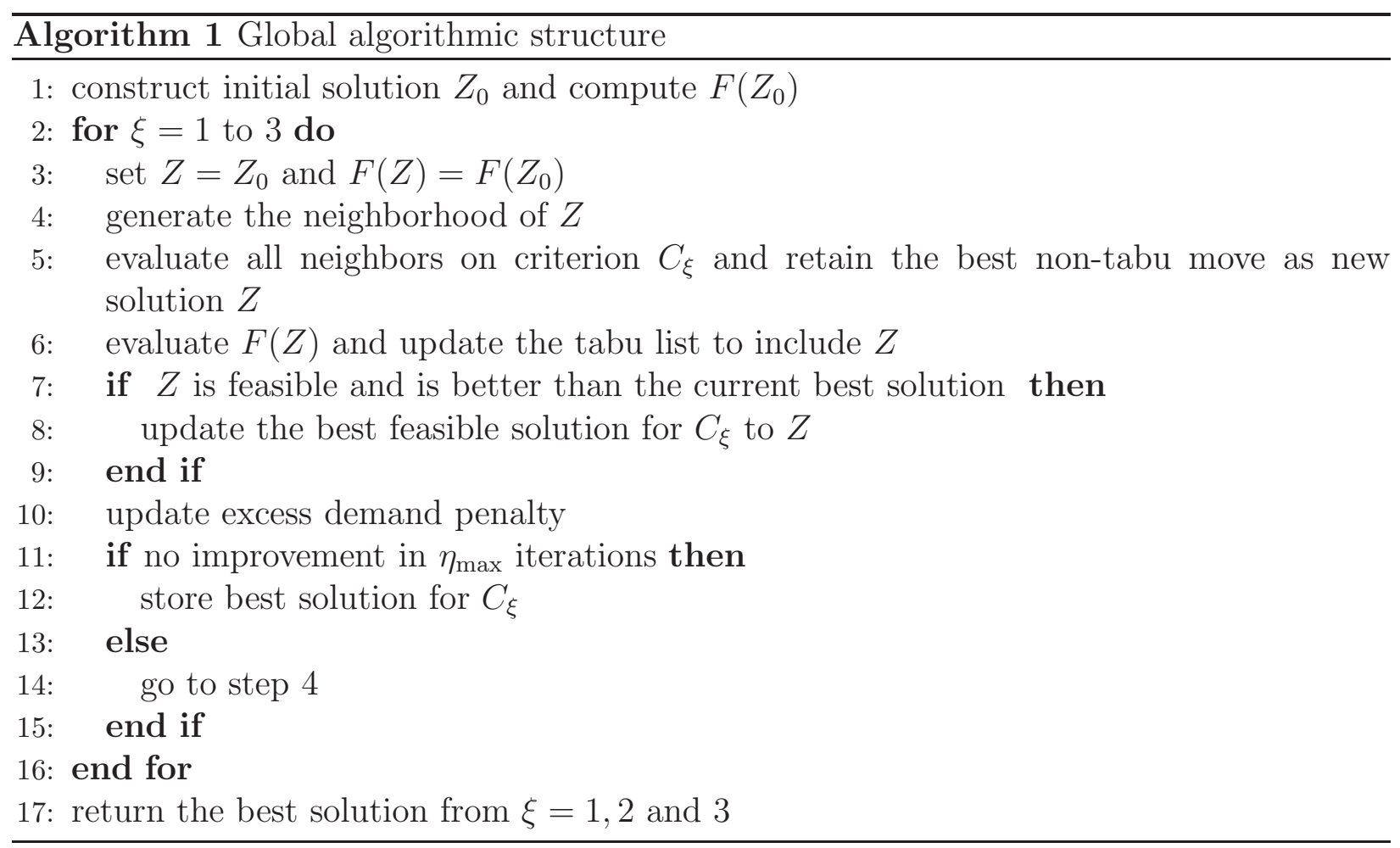

$C_{1}$ - distance based This heuristic is based purely on minimizing the modified travel costs $\Omega_{2}(\cdot)$, i. e., it does not take into account the time windows and their associated penalties, nor does it consider overtime. Thus, $C_{1}$ is similar to the criteria used in local search for the VRP. Let $Z^{\prime}$ be a neighbor of the current solution $Z$ and define $\Delta_{1}\left(Z^{\prime}\right)=\Omega_{2}(Z)-\Omega_{2}\left(Z^{\prime}\right)$. The chosen move is one that is not tabu and maximizes $\Delta_{1}(\cdot)$.

$C_{2}$ - distance based and marginal penalties This measure adds to $C_{1}$ an assessment of the penalty component $\sum_{R_{r} \in Z} \Theta\left(R_{r}\right)$. For given $Z$, the marginal penalty of route $R_{r}$ is $\frac{\Theta\left(R_{r}\right)}{n_{r}+1}$. Consider a move involving two routes $R_{1}$ and $R_{2}$, leading to solution $Z^{\prime}$. Let $n_{1}$ and $n_{2}$ be the number of nodes visited by routes $R_{1}$ and $R_{2}$, respectively, in the current solution $Z$, and $n_{1}^{\prime}$ and $n_{2}^{\prime}$ the number of nodes visited by routes $R_{1}$ and $R_{2}$ in the new solution $Z^{\prime} . C_{2}$ picks the move that is not tabu and maximizes the following 
expression:

$$
\Delta_{2}\left(Z^{\prime}\right)=\Omega_{2}(Z)-\Omega_{2}\left(Z^{\prime}\right)+\rho\left[\Theta\left(R_{1}\right)+\Theta\left(R_{2}\right)-\frac{\Theta\left(R_{1}\right)}{n_{1}+1}\left(n_{1}^{\prime}+1\right)-\frac{\Theta\left(R_{2}\right)}{n_{2}+1}\left(n_{2}^{\prime}+1\right)\right] .
$$

The logic behind this evaluation is based on the observation that penalties increase with the number of customers in the route. Decreasing the number of customers in a route with a large penalty value is likely to decrease the total objective value associated with the route.

$C_{3}$ - distance and buffer based As mentioned in Section 3.1, the buffer size between customers $i$ and $i+1$ is $b(i)=s_{i+1}-\left(s_{i}+d_{i}+u_{i+1}\right)$. Criterion $C_{3}$ favors moves with small buffers. Each buffer unit is penalized by $\gamma$. For each candidate solution $Z^{\prime}$ involving a move between customer $i$ and customer $j$, we compute the following quantity:

$$
\Delta_{3}\left(Z^{\prime}\right)=\Omega_{2}(Z)-\Omega_{2}\left(Z^{\prime}\right)-\gamma[b(i)+b(j)] .
$$

We chose a move that is not tabu and that maximizes $\Delta_{3}(\cdot)$. The reasoning involved in this move selection process is the following: improvements in travel times are more likely to also decrease the penalties when the buffers are small.

Different aspects of the problem are tackled by each criterion. The impact of a move on the travel time $\Omega_{2}(Z)$ is efficiently computed. The accurate impact of a move on the penalty component $\sum_{R_{r} \in Z} \Theta\left(R_{r}\right)$ of the target function, on the other hand, requires evaluation of the SITW model for the affected route or routes. Criteria 2 and 3 attempt to assess moves based on the penalty values of the current solution rather than via the LP model. We note that $C_{2}$ is equivalent to $C_{1}$ for moves involving a single route, which can occur only with Or-opt moves. 


\section{Computational experiments}

We have run a number of experiments to assess the computational performance of our algorithm and to compare the outcomes of the VRP-SITW with both the results of the VRP and of the VRPTW. Throughout this section, the travel cost $c$ in $\Omega(Z)$ is set to one, thus we use the terms distance and travel time interchangeably. For an instance with $N$ nodes, for each customer the $\eta=\lceil 0.3 N\rceil$ closest customers are candidates for a move. The tenure size $\kappa$ is set to 20 . The infeasibility penalty $w$ equals 12 , with $\phi=5$ and $\nu=\frac{3}{4}$. The penalties associated with $C_{2}$ and $C_{3}$ are chosen as $\rho=1$ and $\gamma=0.1$, respectively. The overtime penalty $b$ takes the value 2 . The probability $p_{i}$ is set to one over the total number of legs in a solution. Given a solution with $k$ vehicles, where $k \leq K, p_{i}=\frac{1}{N+k}$. Hence, the probability of disruption is identical for all the legs in the solution.

We consider four disruption scenarios for each leg: $\left|\Psi_{i}\right|=4$. The probabilities of disruption are also the same for each leg $i$, namely $g_{i 1}=0.5, g_{i 2}=0.3, g_{i 3}=0.1$ and $g_{i 4}=0.1$ Finally, the disruption lengths between customers $i$ and $j$ are assumed proportional to the baseline duration $d_{i j}$, namely $l_{i 1}=0.1 d_{i j}, l_{i 2}=0.2 d_{i j}, l_{i 3}=0.5 d_{i j}$ and $l_{i 3}=d_{i j}$.

All experiments are performed on a Intel(R)Core Duo with $2.40 \mathrm{GHz}$ and $2 \mathrm{~GB}$ of RAM. The implementation is coded in $\mathrm{C}++$. The LP instances are solved by embedding Gurobi Optimizer 2.0.2, which uses the simplex algorithm. The reported run times are in seconds. We have adopted two datasets from the literature. The first dataset contains a number of VRP instances from Augerat et al. (1998). We work with 27 VRP instances, with the number of customers ranging from 31 to 79 . The vehicle capacity $Q$ is 100 units. The baseline service time $u_{i}$ for each customer $i$ is set to 10 minutes. The shift start time and end time $s_{s}$ and $s_{e}$ are chosen as zero and 200, respectively. The window length $W_{i}$ equals 60 for all $i$. The second dataset contains VRPTW instances and stems from Solomon (1987). We consider 29 instances with 100 customers (sets R1 (random), C1 (clustered) and RC1 (random and clustered)). The baseline service times $u_{i}$ and window sizes $W_{i}$ are given. The opening hours of the depot are used to determine the shift's starting time $s_{s}$ and ending time $s_{e}$. The 
vehicle capacity $Q$ is 200 units.

Below, we first conduct some experiments related to move selection and tardiness choices (in Section 4.1 and 4.2, respectively), followed by comparisons with VRP (Section 4.3) and with VRPTW (Section 4.4).

\subsection{Move selection}

Table 1 shows the results of implementations for the Augerat instances in which only one of the three criteria $C_{1}, C_{2}$ and $C_{3}$ is used during the optimization; the tardiness penalty $t_{i}=5$ for all arcs. The left side of the table displays the target function value $F(Z)$ attained. The right side of the table exhibits the run time for each of the three measures. We observe that $C_{3}$ outperforms the other two critera in 15 out of the 27 instances, while $C_{1}$ and $C_{2}$ do so in seven and five instances, respectively. On average, $C_{1}$ requires less runtime than $C_{2}$ and $C_{3}$. The average run time over all heuristics is 17.3 minutes. Since we are working in an $a$ priori setting, these running times are acceptable.

Table 2 contains similar results for the Solomon instances. The computation times are larger than those for the first dataset. This is partly due to a greater number of customers, but more importantly the number of customers per route is also larger than before. Thus, the LP subroutine will consume considerably more time. We note that we obtain identical results for some of the instances, which is due to the fact that the time window constraints in these VRPTW instances are now relaxed, and some of instances have the same time window lengths and customer locations. In line with Table 1, the three move selection criteria differ in performance. $C_{2}$ performs best in 23 out of the 27 instances, while this occurs for $C_{1}$ and $C_{3}$ in two and four instances, respectively.

\subsection{Tardiness penalty choices}

In order to evaluate the effects of varying delay penalty costs $t_{i}$, we have conducted experiments under four different cost settings, which are subsequently referred to as 'P5', 'P10', 


\begin{tabular}{lrrrrrrr}
\hline Instance & \multicolumn{3}{c}{ Objective value } & \multicolumn{4}{c}{ CPU time } \\
& \multicolumn{1}{c}{$C_{1}$} & \multicolumn{1}{c}{$C_{2}$} & \multicolumn{1}{c}{$C_{3}$} & \multicolumn{1}{c}{$C_{1}$} & \multicolumn{1}{c}{$C_{2}$} & \multicolumn{1}{c}{$C_{3}$} & Total \\
\hline $32 \mathrm{k} 5$ & 955.4 & 1038.2 & 957.2 & 734 & 103 & 1568 & 2405 \\
$33 \mathrm{k} 5$ & 744.8 & 724.1 & 716.6 & 78 & 121 & 166 & 365 \\
$33 \mathrm{k} 6$ & 801.1 & 798.7 & 791.0 & 213 & 151 & 177 & 541 \\
$34 \mathrm{k} 5$ & 867.9 & 876.1 & 852.7 & 335 & 135 & 374 & 844 \\
$36 \mathrm{k} 5$ & 958.0 & 990.1 & 950.5 & 552 & 222 & 438 & 1212 \\
$37 \mathrm{k} 5$ & 765.6 & 811.8 & 798.6 & 338 & 394 & 210 & 942 \\
$37 \mathrm{k} 6$ & 1071.1 & 1069.0 & 1080.5 & 112 & 158 & 148 & 418 \\
$38 \mathrm{k} 5$ & 822.6 & 832.5 & 823.4 & 361 & 299 & 227 & 887 \\
$39 \mathrm{k} 5$ & 1013.1 & 957.5 & 995.9 & 200 & 302 & 289 & 791 \\
$39 \mathrm{k} 6$ & 963.0 & 956.1 & 952.7 & 184 & 130 & 151 & 465 \\
$44 \mathrm{k} 6$ & 1102.9 & 1057.7 & 1054.7 & 128 & 124 & 175 & 427 \\
$45 \mathrm{k} 6$ & 1078.0 & 2685.8 & 1096.4 & 1117 & 71 & 1142 & 2330 \\
$45 \mathrm{k} 7$ & 1294.4 & 1281.4 & 1302.8 & 80 & 86 & 82 & 248 \\
$46 \mathrm{k} 7$ & 1072.5 & 1059.0 & 1008.7 & 99 & 221 & 401 & 721 \\
$48 \mathrm{k} 7$ & 1256.3 & 1243.1 & 1247.2 & 169 & 230 & 224 & 623 \\
$53 \mathrm{k} 7$ & 1185.3 & 1194.7 & 1165.3 & 192 & 1046 & 376 & 1614 \\
$54 \mathrm{k} 7$ & 1293.7 & 1396.7 & 1335.5 & 253 & 446 & 320 & 1019 \\
$55 \mathrm{k} 9$ & 1158.7 & 1137.4 & 1132.2 & 340 & 212 & 255 & 807 \\
$60 \mathrm{k} 9$ & 1509.2 & 1489.4 & 1473.8 & 108 & 112 & 177 & 397 \\
$61 \mathrm{k} 9$ & 1197.9 & 1239.7 & 1177.3 & 225 & 224 & 214 & 663 \\
$62 \mathrm{k} 8$ & 1509.7 & 1516.0 & 1499.5 & 295 & 893 & 386 & 1574 \\
$63 \mathrm{k} 10$ & 1556.2 & 1411.1 & 1493.0 & 157 & 607 & 292 & 1056 \\
$63 \mathrm{k} 9$ & 1834.5 & 1897.8 & 1840.8 & 343 & 317 & 712 & 1372 \\
$64 \mathrm{k} 9$ & 1658.5 & 1626.5 & 1587.8 & 202 & 431 & 521 & 1154 \\
$65 \mathrm{k} 9$ & 1319.7 & 1307.3 & 1293.2 & 137 & 1249 & 115 & 1501 \\
$69 \mathrm{k} 9$ & 1254.5 & 1276.8 & 1291.3 & 616 & 452 & 552 & 1620 \\
$80 \mathrm{k} 10$ & 2095.0 & 2057.7 & 2046.5 & 399 & 1002 & 693 & 2094 \\
\hline Average & & & & 295 & 361 & 385 & 1040 \\
\hline
\end{tabular}

Table 1: Comparison of the three move selection criteria for the Augerat instances

'Prob' and '1.3dist'. In P5, we choose $t_{i}=5, \forall i \in V \backslash\{0\}$ (which was the choice also in Section 4.1), while P10 corresponds to $t_{i}=10$. Under setting Prop, the delay cost for each customer equals the quantity ordered, so $t_{i}=q_{i}, \forall i \in V \backslash\{0\}$, which represents a situation where the delay penalty is proportional to the demand. The final experimental setting, denoted by 1.3 dist, puts $t_{i}$ equal to 5 for all customers, similarly to P5, but all distances are now increased by $30 \%$. In this way, there is less slack time available, leading to less buffer time to be allocated and resulting in tighter instances.

Table 3 summarizes the results for the four experimental settings after running the full tabu search procedure (with the three criteria combined). The left side of the table shows the achieved target function values. Value $M\left(C_{i}\right)$ denotes the number of times (out of 27) 


\begin{tabular}{lrrrrrrr}
\hline Instance & \multicolumn{3}{c}{ Objective value } & \multicolumn{4}{c}{ CPU time } \\
& \multicolumn{1}{c}{$C_{1}$} & \multicolumn{1}{c}{$C_{2}$} & \multicolumn{1}{c}{$C_{3}$} & \multicolumn{1}{c}{$C_{1}$} & \multicolumn{1}{c}{$C_{2}$} & \multicolumn{1}{c}{$C_{3}$} & Total \\
\hline R101 & 918.6 & 905.7 & 922.4 & 1773 & 3388 & 775 & 5936 \\
R102 & 918.2 & 922.5 & 922.1 & 1724 & 1860 & 769 & 4353 \\
R103 & 918.2 & 922.5 & 922.1 & 1734 & 1865 & 774 & 4373 \\
R104 & 917.2 & 917.0 & 920.5 & 1737 & 3445 & 771 & 5953 \\
R105 & 917.0 & 908.8 & 920.1 & 1722 & 2412 & 767 & 4901 \\
R106 & 917.0 & 908.8 & 920.1 & 1743 & 2384 & 761 & 4888 \\
R107 & 917.0 & 908.8 & 920.1 & 1752 & 2362 & 773 & 4887 \\
R108 & 917.0 & 908.8 & 920.1 & 1765 & 2392 & 764 & 4921 \\
R109 & 917.0 & 908.8 & 920.1 & 1728 & 2375 & 767 & 4870 \\
R110 & 917.0 & 908.8 & 920.1 & 1730 & 2240 & 761 & 4731 \\
R111 & 917.0 & 908.8 & 920.1 & 1717 & 2229 & 768 & 4714 \\
R112 & 917.0 & 908.8 & 920.1 & 1743 & 2240 & 761 & 4744 \\
C101 & 834.7 & 834.6 & 859.2 & 805 & 3209 & 1315 & 5329 \\
C102 & 834.7 & 834.6 & 859.2 & 802 & 3181 & 1328 & 5311 \\
C103 & 834.7 & 834.6 & 859.2 & 799 & 3210 & 1317 & 5326 \\
C104 & 834.7 & 834.6 & 859.2 & 807 & 3271 & 1324 & 5402 \\
C105 & 834.7 & 834.6 & 859.2 & 796 & 3308 & 1317 & 5421 \\
C106 & 834.7 & 834.6 & 859.2 & 799 & 3296 & 1316 & 5411 \\
C107 & 834.7 & 834.6 & 859.2 & 798 & 3211 & 1327 & 5336 \\
C108 & 834.7 & 834.6 & 859.2 & 803 & 3187 & 1319 & 5309 \\
C109 & 834.7 & 834.6 & 859.2 & 792 & 3198 & 1327 & 5317 \\
RC101 & 1024.5 & 1013.2 & 1022.6 & 1198 & 1318 & 1071 & 3587 \\
RC102 & 1024.5 & 1013.2 & 1022.6 & 1196 & 1318 & 1075 & 3589 \\
RC103 & 1024.5 & 1013.4 & 1022.6 & 1195 & 1740 & 1121 & 4056 \\
RC104 & 1024.5 & 1042.0 & 1022.6 & 1201 & 1026 & 1137 & 3364 \\
RC105 & 1025.0 & 1013.6 & 1023.2 & 1195 & 1318 & 1093 & 3606 \\
RC106 & 1024.5 & 1042.0 & 1022.6 & 1189 & 1024 & 1083 & 3296 \\
RC107 & 1024.5 & 1042.0 & 1022.6 & 1209 & 1021 & 1090 & 3320 \\
RC108 & 1024.5 & 1042.0 & 1022.6 & 1191 & 1023 & 1083 & 3297 \\
\hline Average & & & & 1298 & 2347 & 1029 & 4674 \\
\hline
\end{tabular}

Table 2: Comparison of the three move selection criteria for the Solomon instances

that criterion $C_{i}$ produces the best result; these values are presented in the last three lines of the table. Measure $C_{3}$ performs best in more instances in all four experimental settings. The best result for $C_{3}$ is in P5. In total, $C_{2}$ and $C_{3}$ perform best in 30 and 26 instances, respectively, when considering all four experimental settings. The fact that $C_{3}$ accounts for buffer sizes between customers might explain its superior performance.

On average, the objective values for P10 are only $0.7 \%$ higher than for P5. This means that even doubling the customer delay penalty does not affect the final objective value to a large extent. With varying penalties, as in the Prop setting, the values are not dramatically different either. For the case of 1.3 dist, the average objective increase is $36.1 \%$ compared to 


\begin{tabular}{|c|c|c|c|c|c|c|c|c|}
\hline \multirow[t]{2}{*}{ Instance } & \multicolumn{4}{|c|}{ Objective } & \multicolumn{4}{|c|}{ Penalty ratio } \\
\hline & P5 & P10 & Prop & $1.3 \mathrm{dist}$ & P5 & P10 & Prop & 1.3dist \\
\hline $32 \mathrm{k} 5$ & 955.4 & 961.7 & 956.6 & 1290.8 & $16.6 \%$ & $17.1 \%$ & $16.7 \%$ & $18.5 \%$ \\
\hline $33 \mathrm{k} 5$ & 716.6 & 716.9 & 716.5 & 998.7 & $6.3 \%$ & $5.0 \%$ & $6.3 \%$ & $12.4 \%$ \\
\hline $33 \mathrm{k} 6$ & 791.0 & 796.8 & 797.6 & 1066.9 & $5.0 \%$ & $5.7 \%$ & $5.8 \%$ & $8.9 \%$ \\
\hline $34 \mathrm{k} 5$ & 852.7 & 857.6 & 857.2 & 1190.6 & $7.0 \%$ & $7.6 \%$ & $7.6 \%$ & $13.4 \%$ \\
\hline $36 \mathrm{k} 5$ & 950.5 & 960.3 & 957.8 & 1285.6 & $13.5 \%$ & $14.4 \%$ & $14.2 \%$ & $17.8 \%$ \\
\hline $37 \mathrm{k} 5$ & 765.6 & 766.8 & 766.4 & 1101.0 & $10.9 \%$ & $11.0 \%$ & $11.0 \%$ & $17.0 \%$ \\
\hline $37 \mathrm{k} 6$ & 1069.0 & 1079.4 & 1079.3 & 1457.3 & $9.2 \%$ & $9.6 \%$ & $9.6 \%$ & $13.3 \%$ \\
\hline $38 \mathrm{k} 5$ & 822.6 & 824.3 & 824.0 & 1162.0 & $7.6 \%$ & $7.8 \%$ & $7.8 \%$ & $15.2 \%$ \\
\hline $39 \mathrm{k} 5$ & 957.5 & 971.6 & 969.4 & 1283.2 & $11.2 \%$ & $11.6 \%$ & $11.4 \%$ & $15.3 \%$ \\
\hline $39 \mathrm{k} 6$ & 952.7 & 957.8 & 953.4 & 1295.1 & $10.5 \%$ & $11.0 \%$ & $8.7 \%$ & $16.0 \%$ \\
\hline $44 \mathrm{k} 6$ & 1054.7 & 1059.6 & 1059.2 & 1489.6 & $8.8 \%$ & $9.3 \%$ & $9.2 \%$ & $13.4 \%$ \\
\hline $45 \mathrm{k} 6$ & 1078.0 & 1081.3 & 1066.1 & 1469.0 & $8.4 \%$ & $8.6 \%$ & $8.2 \%$ & $12.7 \%$ \\
\hline $45 \mathrm{k} 7$ & 1281.4 & 1298.3 & 1277.7 & 1713.5 & $7.7 \%$ & $8.9 \%$ & $8.5 \%$ & $11.3 \%$ \\
\hline $46 \mathrm{k} 7$ & 1008.7 & 1007.5 & 1009.4 & 1371.5 & $7.0 \%$ & $6.9 \%$ & $7.1 \%$ & $10.6 \%$ \\
\hline $48 \mathrm{k} 7$ & 1243.1 & 1244.1 & 1231.5 & 1662.6 & $10.2 \%$ & $9.0 \%$ & $8.2 \%$ & $11.8 \%$ \\
\hline $53 \mathrm{k} 7$ & 1165.3 & 1168.2 & 1167.1 & 1542.7 & $7.4 \%$ & $7.6 \%$ & $7.5 \%$ & $11.7 \%$ \\
\hline $54 \mathrm{k} 7$ & 1293.7 & 1302.2 & 1302.5 & 1799.6 & $7.6 \%$ & $8.2 \%$ & $8.2 \%$ & $12.3 \%$ \\
\hline $55 \mathrm{k} 9$ & 1132.2 & 1135.5 & 1136.8 & 1506.1 & $2.7 \%$ & $2.9 \%$ & $3.0 \%$ & $5.1 \%$ \\
\hline $60 \mathrm{k} 9$ & 1473.8 & 1482.7 & 1485.1 & 1980.8 & $5.5 \%$ & $5.1 \%$ & $6.2 \%$ & $8.0 \%$ \\
\hline $61 \mathrm{k} 9$ & 1177.3 & 1178.6 & 1178.5 & 1651.2 & $3.4 \%$ & $3.6 \%$ & $3.5 \%$ & $6.6 \%$ \\
\hline $62 \mathrm{k} 8$ & 1499.5 & 1505.7 & 1486.1 & 1986.3 & $9.4 \%$ & $9.7 \%$ & $8.7 \%$ & $11.9 \%$ \\
\hline $63 \mathrm{k} 10$ & 1411.1 & 1500.0 & 1501.2 & 1914.9 & $4.0 \%$ & $4.1 \%$ & $4.2 \%$ & $6.8 \%$ \\
\hline $63 \mathrm{k} 9$ & 1834.5 & 1847.7 & 1844.1 & 2472.9 & $8.5 \%$ & $9.2 \%$ & $9.0 \%$ & $11.3 \%$ \\
\hline $64 \mathrm{k} 9$ & 1587.8 & 1598.7 & 1597.1 & 2166.2 & $8.5 \%$ & $9.1 \%$ & $9.0 \%$ & $11.3 \%$ \\
\hline $65 \mathrm{k} 9$ & 1293.2 & 1295.3 & 1293.7 & 1720.5 & $3.0 \%$ & $3.2 \%$ & $3.1 \%$ & $6.4 \%$ \\
\hline $69 \mathrm{k} 9$ & 1254.5 & 1256.6 & 1256.7 & 1643.9 & $4.0 \%$ & $4.1 \%$ & $4.1 \%$ & $6.4 \%$ \\
\hline $80 \mathrm{k} 10$ & 2046.5 & 2061.4 & 2042.8 & 2756.5 & $9.9 \%$ & $10.6 \%$ & $9.1 \%$ & $11.7 \%$ \\
\hline Average penalty \% & & & & & $7.9 \%$ & $8.2 \%$ & $8.0 \%$ & $11.7 \%$ \\
\hline$M\left(C_{1}\right)$ & 7 & 8 & 7 & 8 & & & & \\
\hline$M\left(C_{2}\right)$ & 5 & 5 & 7 & 9 & & & & \\
\hline$M\left(C_{3}\right)$ & 15 & 14 & 13 & 10 & & & & \\
\hline
\end{tabular}

Table 3: Results for the Augerat instances with four different penalty settings

P5, while the distances are raised by only $30 \%$. This difference can be explained by the fact that when distances rise, there is less buffer time to be allocated and the solutions are more prone to suffer overtime and delay penalties.

The right part of Table 3 shows the 'penalty ratio', which is the proportion

$$
\sum_{R_{r} \in Z} \frac{\Theta\left(R_{r}\right)}{F(Z)}
$$

of the total objective that corresponds to penalties. The average over all four experimental conditions is $9.0 \%$. The lowest ratios are achieved for P5 and Prop, followed by P10, and 
the ratios for 1.3 dist are by far the largest. We conclude that an increase in the distances has a substantial impact on the delay penalties.

\subsection{VRP-SITW versus VRP}

The addition of SITW to the VRP can be expected to affect the distance traveled and the number of vehicles used. To assess the effect, we compare the total distance in VRP-SITW with the optimal VRP solutions (taken from Ralphs (2010)). The details are provided in Table 4. For P5 and P10, the average distance increase is $3.3 \%$ and 3.7\%, respectively, which shows that, at least as far as distance minimization is concerned, our heuristic solutions are rather close to optimal; the same observation can be made for Prop. For 1.3dist the VRP distances are scaled by a factor of 1.3 , but this does not have an important impact on the distance increase. Overall, we conclude that the distance increase is not substantial for any of the settings.

\subsection{VRP-SITW versus VRPTW}

The goal of this section is to evaluate the benefits of the flexibility in setting time windows compared to exogenously predetermined time windows. To this aim, we work with 29 VRPTW instances from Solomon (1987). We compare the results of the VRP-SITW with the best-known solutions for the Solomon instances as reported in Solomon (2010).

Table 5 reports the results. For brevity we denote the travel time, which is equivalent to the distance, by $T_{F}$ for the VRPTW (which has fixed time windows) and by $T_{S}$ for the VRP-SITW. The number of vehicles required in the VRPTW is represented by $K_{F}$ while the number of vehicles used by the VRP-SITW solution is written as $K_{S}$. The third column in Table 5 gives the ratio of the total travel times in both solutions. We observe that the VRP-SITW substantially reduces the travel time for instances with tight time windows such as those in the $\mathrm{R} 1$ and $\mathrm{RC} 1$ sets. Set $\mathrm{C} 1$, on the other hand, achieves zero penalty values, which can be read from the last column of the table. We conclude that these instances 


\begin{tabular}{lcccc}
\hline Instance & \multicolumn{4}{c}{ Increase in distance } \\
\hline & P5 & P10 & Prop & 1.3 dist \\
\hline 32 k5 & $101.1 \%$ & $101.1 \%$ & $102.7 \%$ & $101.1 \%$ \\
33 k5 & $101.3 \%$ & $102.7 \%$ & $101.6 \%$ & $101.3 \%$ \\
33 k6 & $101.2 \%$ & $101.2 \%$ & $100.7 \%$ & $101.2 \%$ \\
34 k5 & $101.5 \%$ & $101.4 \%$ & $101.5 \%$ & $101.4 \%$ \\
36 k5 & $102.5 \%$ & $102.5 \%$ & $101.3 \%$ & $102.5 \%$ \\
37 k5 & $101.4 \%$ & $101.4 \%$ & $104.5 \%$ & $101.4 \%$ \\
37 k6 & $102.0 \%$ & $102.5 \%$ & $102.1 \%$ & $102.5 \%$ \\
38 k5 & $103.5 \%$ & $103.5 \%$ & $103.3 \%$ & $103.5 \%$ \\
39 k5 & $102.6 \%$ & $103.7 \%$ & $100.9 \%$ & $103.7 \%$ \\
39 k6 & $102.3 \%$ & $102.3 \%$ & $100.5 \%$ & $104.5 \%$ \\
44 k6 & $102.4 \%$ & $102.4 \%$ & $105.6 \%$ & $102.4 \%$ \\
45 k6 & $104.6 \%$ & $104.6 \%$ & $104.5 \%$ & $103.6 \%$ \\
45 k7 & $103.1 \%$ & $103.1 \%$ & $101.9 \%$ & $101.9 \%$ \\
46 k7 & $102.1 \%$ & $102.1 \%$ & $102.7 \%$ & $102.1 \%$ \\
48 k7 & $103.9 \%$ & $105.4 \%$ & $105.0 \%$ & $105.2 \%$ \\
53 k7 & $106.5 \%$ & $106.5 \%$ & $103.4 \%$ & $106.5 \%$ \\
54 k7 & $102.0 \%$ & $102.0 \%$ & $103.6 \%$ & $102.0 \%$ \\
55 k9 & $102.6 \%$ & $102.6 \%$ & $102.4 \%$ & $102.6 \%$ \\
60 k9 & $102.7 \%$ & $103.8 \%$ & $103.4 \%$ & $102.7 \%$ \\
61 k9 & $109.4 \%$ & $109.4 \%$ & $114.2 \%$ & $109.4 \%$ \\
62 k8 & $105.0 \%$ & $105.0 \%$ & $104.0 \%$ & $104.9 \%$ \\
63 k10 & $103.1 \%$ & $109.5 \%$ & $104.5 \%$ & $109.5 \%$ \\
63 k9 & $103.4 \%$ & $103.4 \%$ & $104.0 \%$ & $103.4 \%$ \\
64 k9 & $103.7 \%$ & $103.7 \%$ & $105.6 \%$ & $103.7 \%$ \\
65 k9 & $106.1 \%$ & $106.1 \%$ & $104.8 \%$ & $106.1 \%$ \\
69 k9 & $103.3 \%$ & $103.3 \%$ & $101.5 \%$ & $103.3 \%$ \\
80 k10 & $104.4 \%$ & $104.4 \%$ & $106.0 \%$ & $105.1 \%$ \\
\hline Average & $103.3 \%$ & $103.7 \%$ & $103.5 \%$ & $103.6 \%$ \\
\hline
\end{tabular}

Table 4: Comparison of VRP-SITW with optimal VRP solutions for the Augerat instances

have quite unrestrictive time windows and exhibit a behavior similar to the VRP instances studied in Section 4.3. Across the datasets, the penalty component $\sum_{R_{r} \in Z} \Theta\left(R_{r}\right)$ comprises at most $6.3 \%$ of the total objective value.

The fifth column of Table 5 displays the number of vehicles saved in VRP-SITW compared to VRPTW. A substantial reduction in the required number of vehicles is observed in the $\mathrm{R} 1$ and $\mathrm{RC} 1$ sets. In set $\mathrm{C} 1$, however, no such reduction is achieved. We conclude that those instances that allow for substantial reductions in travel times are eligible for similar improvements with respect to the number of vehicles. 


\begin{tabular}{lrrrrr}
\hline Instance & \multicolumn{1}{c}{$T_{F}$} & $T_{S} / T_{F}$ & $K_{F}$ & $K_{F}-K_{S}$ & $\sum_{R_{r} \in Z} \Theta\left(R_{r}\right) / F(Z)$ \\
\hline R101 & 1637.7 & $52.0 \%$ & 20 & 12 & $6.3 \%$ \\
R102 & 1466.6 & $59.9 \%$ & 18 & 10 & $4.5 \%$ \\
R103 & 1208.7 & $72.7 \%$ & 14 & 6 & $4.5 \%$ \\
R104 & 971.5 & $89.7 \%$ & 11 & 3 & $5.2 \%$ \\
R105 & 1355.3 & $63.5 \%$ & 15 & 7 & $5.6 \%$ \\
R106 & 1251.98 & $68.7 \%$ & 12 & 4 & $5.6 \%$ \\
R107 & 1064.6 & $80.8 \%$ & 11 & 3 & $5.6 \%$ \\
R108 & 960.88 & $89.5 \%$ & 9 & 1 & $5.6 \%$ \\
R109 & 1146.9 & $75.0 \%$ & 13 & 5 & $5.6 \%$ \\
R110 & 1068 & $80.5 \%$ & 12 & 4 & $5.6 \%$ \\
R111 & 1048.7 & $82.0 \%$ & 12 & 4 & $5.6 \%$ \\
R112 & 982.14 & $87.6 \%$ & 9 & 1 & $0.0 \%$ \\
C101 & 827.3 & $100.9 \%$ & 10 & 0 & $0.0 \%$ \\
C102 & 827.3 & $100.9 \%$ & 10 & 0 & $0.0 \%$ \\
C103 & 826.3 & $101.0 \%$ & 10 & 0 & $0.0 \%$ \\
C104 & 822.9 & $101.4 \%$ & 10 & 0 & $0.0 \%$ \\
C105 & 827.3 & $100.9 \%$ & 10 & 0 & $0.0 \%$ \\
C106 & 827.3 & $100.9 \%$ & 10 & 0 & $0.0 \%$ \\
C107 & 827.3 & $100.9 \%$ & 10 & 0 & $0.0 \%$ \\
C108 & 827.3 & $100.9 \%$ & 10 & 0 & $1.1 \%$ \\
C109 & 827.3 & $100.9 \%$ & 10 & 0 & $1.1 \%$ \\
RC101 & 1619.8 & $61.9 \%$ & 15 & 6 & $1.4 \%$ \\
RC102 & 1457.4 & $68.8 \%$ & 14 & 5 & $1.7 \%$ \\
RC103 & 1258 & $79.4 \%$ & 13 & 4 & $1.1 \%$ \\
RC104 & 1261.67 & $79.6 \%$ & 11 & 2 & $1.7 \%$ \\
RC105 & 1513.7 & $66.2 \%$ & 15 & 6 & $2.7 \%$ \\
RC106 & 1424.73 & $70.5 \%$ & 11 & 2 & \\
RC107 & 1207.8 & $83.2 \%$ & 12 & 3 & \\
\hline Average & & $82.9 \%$ & & & \\
\hline & & & & & $0.7 \%$ \\
\hline
\end{tabular}

Table 5: Comparison of VRP-SITW with the best known VRPTW solutions for the Solomon instances

\section{Conclusions}

In this paper, we have analyzed the situation of carrier companies that face the problem of making routing decisions combined with the quotation of arrival times to their customers; we have referred to this setting by the term 'Self-Imposed Time Windows' (SITW). In the context of vehicle routing, the resulting VRP-SITW extends the VRP by the incorporation of customer-specific service aspects, reflected in the carrier company's ability to uphold the time windows once quoted, in a stochastic environment. In comparison with the VRP with exogenous time windows (VRPTW), the customer service requirement is somewhat relaxed, in that the service provider has ex ante flexibility in choosing a convenient time interval that 
will be quoted.

Our solution approach is a hybrid algorithm that comprises two main components: routing and scheduling. The routing component is handled via a tabu search procedure, while scheduling is performed by solving an LP model that implicitly inserts buffers into each route's schedule.

We have compared the VRP to VRP-SITW under different choices for penalty structures and distances. The results of our tests indicate that the VRP-SITW requires an average increase of some $3.5 \%$ in distance. Further research might focus on the impact of additional vehicles on the penalties.

Contrary to the VRP, the VRPTW exhibits substantial differences when compared to VRP-SITW. In most cases, the VRP-SITW requires significantly less distance and uses far less vehicles. Clearly, the VRP-SITW benefits greatly from its flexibility in setting the time windows. In our opinion, there is important potential in conducting an in-depth study of various flexibility levels in choosing delivery windows. Such a study can be beneficial, for instance, when negotiating service contracts. Another extension might look into the setting where only a subset of customers have fixed time windows. Furthermore, given some alterations the proposed model can also accommodate driving breaks, by using the buffers for the breaks. The proposed model establishes an a priori plan for a static environment. Yet another major extension of the model might incorporate the quotation of time windows for dynamically arriving orders. Finally, a trade-off may be conjectured between tardiness penalties and total travel times. Additional vehicles, for instance, will tend to improve the ability to uphold time windows but will generally increase travel times. Such trade-offs also offer opportunities for further work. 


\section{References}

Augerat, P., J.M. Belenguer, E. Benavent, A. Corber, D. Naddef. 1998. Separating capacity constraints in the CVRP using tabu search. European Journal of Operational Research $106546-557$.

Ballestín, F., R. Leus. 2008. Meta-heuristics for stable scheduling on a single machine. Computers \& Operations Research 35 2175-2192.

Bräysy, O., M. Gendreau. 2005a. Vehicle routing problem with time windows, part I: Route construction and local search algorithms. Transportation Science 39(1) 104-118.

Bräysy, O., M. Gendreau. 2005b. Vehicle routing problem with time windows, part II: Metaheuristics. Transportation Science 39(1) 119-139.

Daniels, R., J. Carrillo. 1997. $\beta$-robust scheduling for single-machine systems with uncertain processing times. IIE Transactions 29 977-985.

Daniels, R., P. Kouvelis. 1995. Robust scheduling to hedge against processing time uncertainty in single-stage production. Management Science 41 363-376.

Finke, D.A., D.J. Medeiros, M.T. Traband. 2007. Multiple machine JIT scheduling: a tabu search approach. International Journal of Production Research 45(21) 4899-4915.

Flisberg, P., B. Lidéna, M. Rönnqvist. 2009. A hybrid method based on linear programming and tabu search for routing of logging trucks. Computers $\&$ Operations Research $\mathbf{3 6}(4)$ $1122-1144$.

Garcia, B.L., J.Y. Potvin, J.M. Rousseau. 1994. A parallel implementation of the tabu search heuristic for vehicle routing problems with time window constraints. Computers $\mathscr{E}$ Operations Research 21(9) 1025-1033.

Gendreau, M., A. Hertz, G. Laporte. 1994. A tabu search heuristic for the vehicle routing problem. Management Science 40(10) 1276-1290. 
Gendreau, M., G. Laporte, R. Séguin. 1996. A tabu search heuristic for the vehicle routing problem with stochastic demands and customers. Operations Research 44(3) 469-477.

Gendreau, M, G. Laporte, M.M. Solomon. 1995. Single-vehicle routing and scheduling to minimize the number of delays. Transportation Science 29(1) 56-62.

Herroelen, W., R. Leus. 2004. The construction of stable project baseline schedules. European Journal of Operational Research 156 550-565.

Hertz, A., G. Laporte, M. Mittaz. 2000. A tabu search heuristic for the capacitated arc routing problem. Operations Research 48(1) 129-135.

Hopp, W.J., M.L. Spearman. 1996. Factory Physics, Foundations for Manufacturing Management. Mc-Graw Hill.

Jabali, O., T. Van Woensel, A.G. de Kok, C. Lecluyse, H. Peremans. 2009. Time-dependent vehicle routing subject to time delay perturbations. IIE Transactions 41(12) 1049-1066.

Kouvelis, P., R. Daniels, G. Vairaktarakis. 2000. Robust scheduling of a two-machine flow shop with uncertain processing times. IIE Transactions 32 421-432.

Kouvelis, P., G. Yu. 1997. Robust Discrete Optimization and its Applications. Kluwer Academic Publishers.

Laporte, G. 1992. The vehicle routing problem: An overview of exact and approximate algorithms. European Journal of Operational Research 59(3) 345-358.

Laporte, G., F. Louveaux, H. Mercure. 1992. The vehicle routing problem with stochastic travel times. Transportation Science 26(3) 161-170.

Leus, R., W. Herroelen. 2005. The complexity of machine scheduling for stability with a single disrupted job. Operations Research Letters 33 151-156. 
Leus, R., W. Herroelen. 2007. Scheduling for stability in single-machine production systems. Journal of Scheduling $10223-235$.

Li, X., P. Tian, S.C.H Leung. 2010. Vehicle routing problems with time windows and stochastic travel and service times: Models and algorithm. International Journal of Production Economics 125 137-145.

Mitrović-Minć, S., G. Laporte. 2004. Waiting strategies for the dynamic pickup and delivery problem with time windows. Transportation Research Part B 38 635-655.

Or, I. 1976. Traveling salesman-type combinatorial poroblems and their relation to the logistics of regional blood banking. Ph.D. Thesis, Northwestern University, Evanston, Illinois.

Potvin, J.Y., J.M. Rousseau. 1995. An exchange heuristic for routing problems with time windows. Journal of the Operational Research Society 46(12) 1433-1446.

Puchinger, J., G.R. Raidl. 2005. Combining metaheuristics and exact algorithms in combinatorial optimization: A survey and classification. J. Mira, J.R. Álvarez, eds., Artificial Intelligence and Knowledge Engineering Applications: A Bioinspired Approach. Lecture Notes in Computer Science, vol. 3562. Springer, 41-53.

Ralphs, T. 2010. Branch Cut and Price Resource Web. URL http://www. branchandcut.org. Last accessed October 2010.

Solomon, M.M. 1987. Algorithms for the vehicle routing and scheduling problems with time window constraints. Operations Research 35(2) 254-265.

Solomon, M.M. 2010. VRPTW Benchmark Problems. URL http://web.cba.neu.edu/ msolomon/problems.htm. Last accessed April 2010.

Taillard, E., P. Badeau, M. Gendreau, F. Guertin, J.Y. Potvin. 1997. A tabu search heuristic for the vehicle routing problem with soft time windows. Computers $\mathscr{E}$ Operations Research $31(2)$ 170-186. 
Xiang, S., C. Chu, H. Chen. 2008. The study of a dynamic dial-a-ride problem under timedependent and stochastic environments. European Journal of Operational Research 185(2) $534-551$. 